\title{
DETERMINAÇÃO DE METAIS NAS FOLHAS E CHÁS DE PARIRI (Arrabidaea chica), POR ESPECTROSCOPIA DE ABSORÇÃO ATÔMICA, ORIUNDAS DA FEIRA DO VER-O-PESO E DISTRITO DE MOSQUEIRO (Pa)
}

Ana Carolina de Nazaré Gonçalves da Silva ${ }^{1}$; Cristine Bastos do Amarante $^{2}$.

1. Graduada em Licenciatura Plena em Ciências Naturais - Química pela

Universidade do Estado do Pará (UEPa).

carolinagoncalvesjc@gmail.com

2. Doutora em Química. Pesquisadora da Coordenação de Ciências da Terra e Ecologia do Museu Paraense Emílio Goeldi.

Recebido em: 06/04/2019 - Aprovado em: 10/06/2019 - Publicado em: 30/06/2019 DOI: 10.18677/EnciBio_2019A52

\begin{abstract}
O emprego das plantas para desempenhar funções terapêuticas é uma herança de antigas gerações, que sobreviveu ao advento da modernidade e ganhou espaço nos dias atuais. Esse hábito, conquistou a atenção da ciência e logo houve um aumento expressivo em pesquisas sobre a composição química das ervas utilizadas e seus possíveis efeitos curativos. A planta Arrabidaea chica conhecida popularmente como pariri, crajirú, cipó-cruz, dentre outros, de acordo com os ensinamentos empíricos, é muito consumida no interior e região metropolitana de Belém do Pará na forma de chá com finalidades anti-anêmicas, anti-inflamatórias e cicatrizantes. Por esse motivo, o objetivo do presente trabalho é quantificar o teor de Ferro (Fe), Magnésio (Mg), Cálcio, (Ca) Cobre (Cu), Zinco ( $\mathrm{Zn})$ e Manganês (Mn) existentes nas folhas secas e nos chás da erva. As análises foram realizadas no Laboratório de Absorção Atômica do Museu Emílio Goeldi, com amostras em triplicata de chás preparados por infusão e decocção, de folhas de Pariri adquiridas na Feira do Vero-Peso e na llha de Mosqueiro. Segundo os resultados, $\mathrm{Ca}, \mathrm{Mg}, \mathrm{Zn}$ e $\mathrm{Mn}$, foram detectados nas folhas e chás, $\mathrm{Fe}$, apenas nas folhas secas e $\mathrm{Cu}$, não foi detectado. Percebeu-se, que os teores dos elementos, nos chás, estão de acordo com a literatura e com os parâmetros para ingestão diária recomendados pela ANVISA. Dessa forma, confirma-se, por meio da caracterização química, a presença de sais minerais que tornam real a possibilidade de um potencial terapêutico das folhas de Pariri, desde que manuseadas de modo correto e consumidas sem demasia.
\end{abstract}

RESUMO

PALAVRAS-CHAVE: Absorção Atômica. Metais. Pariri.

\section{DETERMINATION OF METALS IN LEAVESAND TEA OFPARIRI (Arrabidaea chica), FROM THE VER-O-PESO FAIR AND MOSQUEIRO (Pa) DISTRICT, BYSPECTROSCOPY OF ATOMIC ABSORPTION}

\footnotetext{
ABSTRACT

The use of plants to develop therapeutic functions is a habit inherited from ancient generations, which survived the advent of modernity and gained space today. This
} 
habit has attracted the attention of science and soon there was an expressive increase in research on the chemical composition of the herbs used and their possible curative effects. The Arrabidaea chica plant popularly known as Pariri, crajirú, cipó-cruz, among others, according to the empirical teachings, it is widely used in the countryside and metropolitan region of Belém-Pará as anti-anemic, antiinflammatory and healing tea. For this reason, the goal of this paper is to quantify the content of Iron (Fe), Magnesium (Mg), Calcium (Ca) Copper (Cu), Zinc ( $\mathrm{Zn}$ ) and Manganese $(\mathrm{Mn})$ in dry leaves and herbal teas of this plant. The analyzes were carried out in the Atomic Absorption Laboratory of the Emílio Goeldi Museum, with triplicate samples of tea prepared by infusion and decoction, from Pariri leaves purchased at the Ver-o-Peso Fair and at Mosqueiro Island. According to the results, $\mathrm{Ca}, \mathrm{Mg}, \mathrm{Zn}$ and $\mathrm{Mn}$, were detected in leaves and tea, Fe, only in dry leaves and $\mathrm{Cu}$, was not detected. It was noticed that the contents of the elements in the teas are in agreement with the literature and with the parameters for daily intake recommended by ANVISA (National Health Surveillance Agency). Thus, the chemical characterization of the presence of mineral salts confirms the possibility of a therapeutic potential of Pariri leaves, provided that they are correctly handled and not consumed in excess.

KEYWORDS: Atomic Absorption. Metals. Pariri.

\section{INTRODUÇÃO}

O uso de ervas como medicamento é realizado há tempos pela humanidade. Durante a idade média, mulheres conhecidas como curandeiras utilizavam variedades de plantas para auxiliar no tratamento de enfermidades, (FREIRE et al., 2006). O emprego das plantas no preparo de chás foi difundido em diversas culturas, considerando que estes apresentam compostos biologicamente ativos, a exemplo de flavonoides, catequinas, vitaminas e sais minerais, que auxiliam no tratamento de diversas doenças (SANTOS et al., 2008).

Hoje, inúmeras pessoas ainda utilizam ervas como primeiro recurso terapêutico. Assim, o interesse ao estudo de constituintes inorgânicos ganhou espaço no meio científico, devido auxiliarem no metabolismo ou, dependendo da sua concentração, poderem ser prejudiciais à saúde do homem (DINIZ et al., 2013).

De acordo com a Organização Mundial da Saúde (OMS), apenas 20\% da população mundial não utiliza plantas medicinais, o que apresenta um cenário positivo quanto ao uso das mesmas (BRASILEIRO et al., 2013), fato ratificado pela Associação Brasileira das Empresas do Setor Fitoterápico, Suplemento Alimentar e de Promoção da Saúde (ABIFISA, 2007), maior parte da população brasileira utiliza alguma planta medicinal, estimulado em $91,9 \%$ dos brasileiros.

O território brasileiro apresenta uma exuberante biodiversidade, resultando em um extenso comércio de plantas medicinais a céu aberto, a exemplo, no Estado do Pará, as ervas vindas das ilhas, são vendidas na maior feira aberta da América Latina, o Ver-o-Peso, dentre elas Arrabidaea chica (AMOROSO; GÉLY, 1988). Espécie correspondente à família Bignoniaceae nativa das florestas tropicais, encontrada na América Central, em toda a Amazônia até o Rio Grande do Sul (PAULETTI et al., 2003).

Conhecida popularmente como pariri (no Pará) ou crajirú (no Amazonas), é uma erva trepadeira, cujas folhas verdes escuras, possuem forma de ponta de lança peciolada, glabos nas duas faces, coriáceos, retículos venosos, incolor ou não, sem cheiro, finas e compridas, as quais floram em três unidades, sobre um cipó, tornando-se avermelhadas quando estão desidratadas, são compostas por pigmentos derivados de genipina (Figura 1), bixina (Figura 2) e crajurina (Figura 3), 
que produzem um corante de cor vermelho-escuro, utilizado no tingimento de diversas fibras artesanais. (SANDWITH ; HUNT, 1974 ;CORRÊA, 1978).

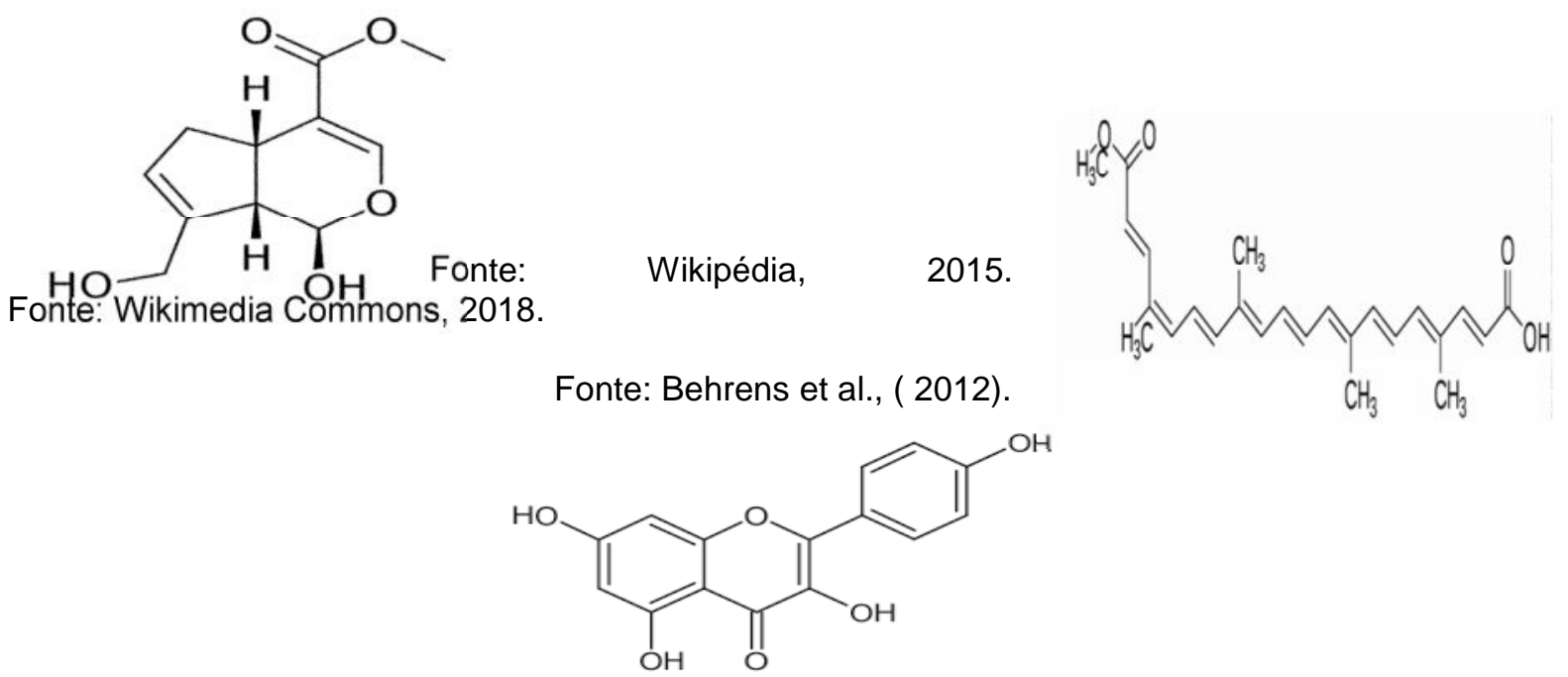

As folhas contêm como princípio ativo o ácido anisíaco, crajurina, taninos, cianocobalamina e ferro assimilável (ALBURQUERQUE, 1989). Segundo Costa e Lima (1989), a planta é largamente utilizada pela população como medicamento, apresentando efeitos cicatrizantes, anti-inflamatórios, anti-anêmicos, combatendo diarreias, doenças hemorrágicas, leucemias, micoses, cólicas intestinais, entre outros.

Para Zorn et al. (2001), os responsáveis pela ação anti-inflamatória são as 3desoxiantocianidinas aliadas à atividade de outros compostos presentes na planta. Segundo Takemura, (1995) ação de antocianinas, flavonoides, taninos, fitosteróis e 7,4- dihidróxi- 5-metoxiflavona (Figura 4), também correspondem ao conjunto de ações que auxiliam no processo de desinflamação. Assim como, a atividade dos pigmentos carajurina (cor vermelha), presentes nos flavonoides e a carajurona (TAKEMURA, 1995).<smiles>COc1cc(O)cc2oc(-c3ccc(O)cc3)cc(=O)c12</smiles>

FIGURA 4: molécula da 7,4- dihidróxi- 5-metoxiflavona Fonte: Behrens et al., 2012.

Conforme Takemura (1995) utiliza-se também na indústria de cosméticos na forma de sabonete antiacne; e remédios antifúngicos, segundo Barbosa e Quignard (1998). Ou ainda, para tratamento da doença púrpura, que está relacionada a um distúrbio autoimune marcado pelo ataque das próprias defesas às plaquetas 
(SPONCHIATO, 2017). As formas de consumo do pariri se fazem por meio do preparo de chás, seja por infusão, maceração, decocção ou por tintura. A administração do consumo é por via oral e/ou por lavagens vaginais (BORRÁS, 2003).

Sobre os sais, o Ferro é um micronutriente atuante no processo de fotossíntese e essencial para hemoglobina (BRITES, 2014). Enquanto o Cálcio é o macroelemento mais abundante no organismo, dentre várias funções, é responsável também pela atividade plaquetária (NUNES, 2013). O Magnésio é o segundo mais abundante cátion intracelular, é responsável pela alteração do tônus vascular, o que pode melhorar o processo inflamatório (CUNHA et al., 2011).O Zinco é conhecido como nutriente do crescimento humano, pois constitui diversas enzimas envolvidas no metabolismo (FÁVARO; VANNUCCHI, 1990).

O Manganês é importante para a estrutura dos ossos, para o sistema nervoso central e para a reprodução. O Cobre é um micromineral essencial para o sangue, sua carência é observada em casos especiais como em lactentes prematuros ou recuperando-se de desnutrição (ANDREWS; SMITH, 2000).

Sobre os locais de análise, a ilha de Mosqueiro de acordo com dados do Instituto Brasileiro de Geografia e Estatística (IBGE, 2018), possui uma área de 212 $\mathrm{Km}^{2}$ e população de aproximadamente 27 mil habitantes. O termo "Mosqueiro", de acordo com Meira Filho (1978), teve início com os termos como, "moqueio" o qual significa uma das formas mais usadas para a preparação de alimentos onde não há recursos para a conservação.

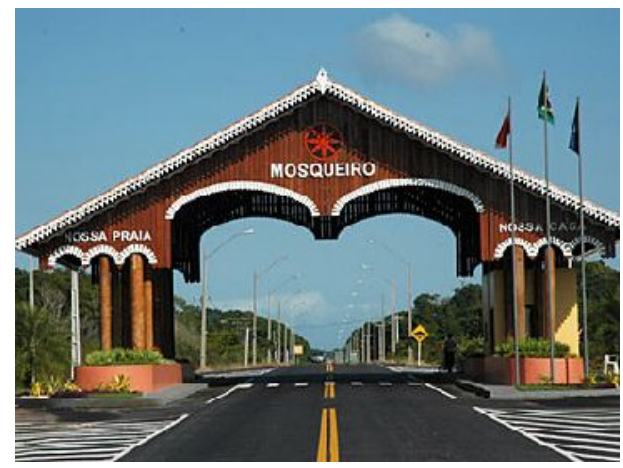

FIGURA 5: Entrada do Distrito de Mosqueiro em Belém do Pará. Fonte: Prefeitura Municipal De Belém, (2015).

Por sua vez, a feira do Ver-o-Peso, localizada às margens da baía do Guajará, foi inaugurada em 1625, o nome do mercado deriva da expressão casa de "Haver o Peso" devido à atividade de verificação do peso das mercadorias para obter o valor dos impostos repassados à coroa portuguesa, no início do século XVII. Atualmente é conceituado como um grande mercado aberto, considerada a maior feira da América Latina, responsável por abastecer Belém do Pará (OLIVEIRA, 2017). 


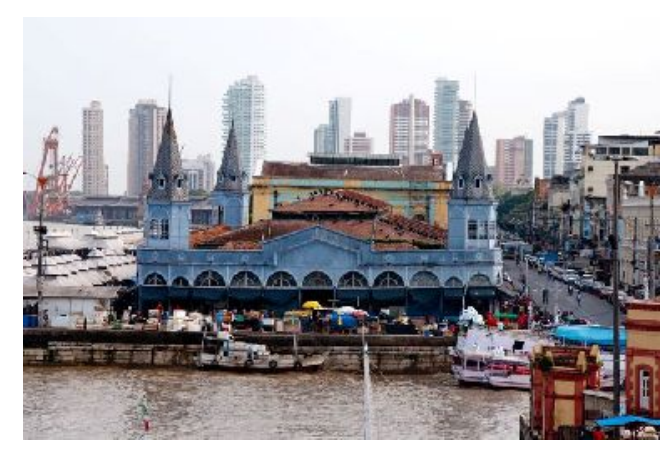

FIGURA 6: Feira do Ver-o-Peso, Belém do Pará.

Fonte: Folha de São Paulo, (2016)

$O$ presente trabalho teve como objetivo determinar $\mathrm{O}$ teor total de sais minerais como: Cálcio ( $\mathrm{Ca})$, Magnésio $(\mathrm{Mg})$, Zinco $(\mathrm{Zn})$, Manganês $(\mathrm{Mn})$, Ferro ( $\mathrm{Fe})$ e Cobre $(\mathrm{Cu})$ presentes nas folhas e chás, infusão e decocção, do pariri, comercializadas no Mercado Ver-o-Peso e coletadas na ilha de Mosqueiro.

\section{Locais de Coleta}

\section{MATERIAIS E MÉTODOS}

As amostras de folhas de pariri foram coletadas em dois locais diferentes: na ilha de Mosqueiro, mais especificamente no Bairro da Vila, cep 66920-000 no horário de 9-10h da manhã e no mercado do Ver-o-Peso, localizado no bairro da Cidade Velha, na Avenida Boulevard Castilhos França, S/N- Comércio, Belém - Pa, cep 66013-030. Ambas as amostras foram adquiridas, no mês de novembro quando se iniciam as chuvas constantes ou o conhecido "inverno paraense".

\section{Preparo das amostras}

As amostras submetidas à digestão foram trituradas e pesadas em uma balança analítica da marca Marte modelo AY220 utilizando uma massa de aproximadamente $0,5 \mathrm{~g}$. Em seguida, digeridas em triplicata, em uma mistura de ácido nitroperclórico $\left(\mathrm{HNO}_{3}\right.$ e $\left.\mathrm{HClO}_{4}\right)$ PA na proporção 3:1 em um bloco digestor a $200^{\circ} \mathrm{C}$. Após a digestão, as amostras foram filtradas e transferidas para balões volumétricos de $50 \mathrm{~mL}$. As amostras foram secas em estufa da marca Fabbe LTDA durante 30 minutos à temperatura de $70 \stackrel{\circ}{\circ}$, em seguida, pulverizadas em moinho analítico da marca Quimis e armazenadas em tubo falcon de $50 \mathrm{~mL}$, adaptado de Assis (2015).

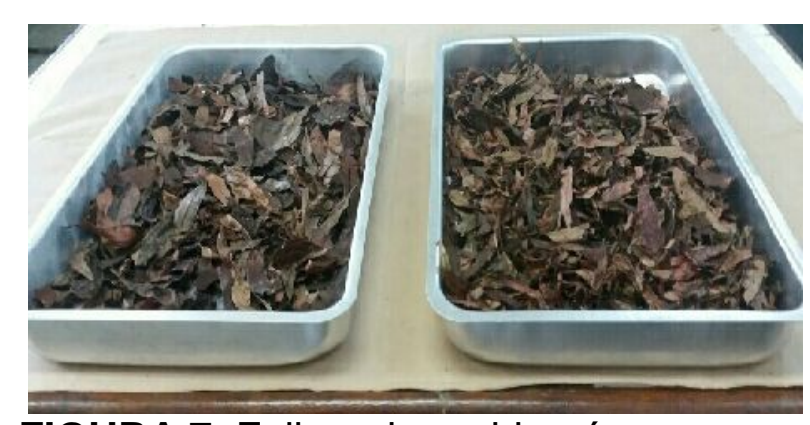

FIGURA 7: Folhas de pariri após secagem.

Fonte: arquivo pessoal ,(2017) 


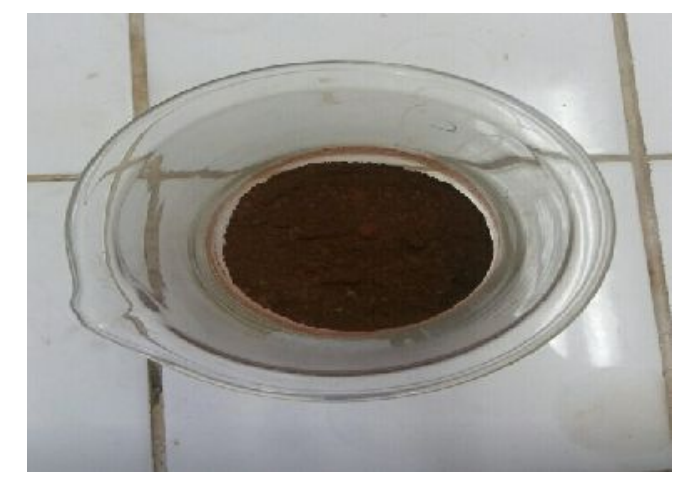

FIGURA 8: Pó das folhas secas após trituração.

Fonte: arquivo pessoal, (2017)

\section{Disponibilidade dos metais nos chás}

Para avaliação da disponibilidade dos metais, as folhas secas da planta foram pesadas em triplicatas em porções de aproximadamente 0,05 $\mathrm{g}$ para os dois locais de procedência. Os chás foram preparados de duas maneiras: infusão, que consiste em ferver a água e despejá-la sobre as folhas da planta, contidas em um becker de $100 \mathrm{~mL}$, por um período de 5 minutos para extração, em seguida filtrada e armazenada em tubos falcon de $50 \mathrm{~mL}$. No preparo por decocção, as plantas secas também armazenadas no becker de $100 \mathrm{~mL}$ foram cozidas em água até $100^{\circ} \mathrm{C}$, esperando-se 5 minutos para extração, em seguida as amostras foram filtradas e armazenadas em tubo falcon $50 \mathrm{~mL}$.

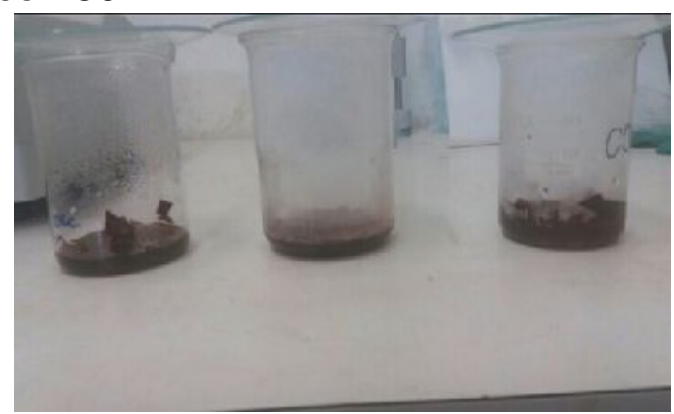

FIGURA 9: Triplicatas de infusos durante os 5 minutos de extração dos minerais.

Fonte: arquivo pessoal, (2017)

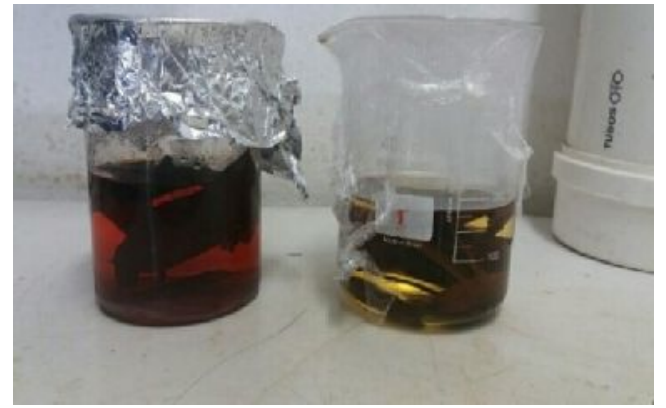

FIGURA 10: Amostras dos chás, decocção à esquerda e infusão à direita.

Fonte: arquivo pessoal, (2017)

\section{Determinação do Teor total}

Os teores de $\mathrm{Fe}$ nos chás foram determinados no Laboratório de Análises Químicas do Museu Paraense Emílio Goeldi. Para determinação do teor dos metais 
foi utilizado um espectrômetro de absorção atômica com chama ar/acetileno, marca Thermo Scientific, modelo iCE 3000. As condições instrumentais para a determinação dos elementos estão apresentadas na tabela 01.

TABELA 1- Parâmetros instrumentais usados na determinação de $\mathrm{Ca}, \mathrm{Mg}, \mathrm{Zn}, \mathrm{Mn}$, $\mathrm{Fe} \mathrm{e} \mathrm{Cu.}$

\begin{tabular}{lccc}
\hline Elemento & $\begin{array}{c}\text { Comprimento de } \\
\text { onda }(\mathbf{n m})\end{array}$ & $\begin{array}{c}\text { Corrente da } \\
\text { da lâmpada }(\%)\end{array}$ & $\begin{array}{c}\text { Resolução } \\
\text { espectral }(\mathbf{n m})\end{array}$ \\
\hline $\mathrm{Ca}$ & 422,7 & 100 & 0,5 \\
$\mathrm{Mg}$ & 285,2 & 75 & 0,5 \\
$\mathrm{Zn}$ & 213,9 & 75 & 0,2 \\
$\mathrm{Mn}$ & 279,5 & 75 & 0,2 \\
$\mathrm{Fe}$ & 248,3 & 75 & 0,2 \\
$\mathrm{Cu}$ & 324,8 & 75 & 0,5 \\
\hline
\end{tabular}

Fonte: Museu Paraense Emílio Goeldi, Laboratório de Absorção Atômica, 2017.

\section{Análise Estatística}

Para quantificação dos elementos nos digeridos e nos chás medidos pelo FAAS (Flame Atomic Absorption Spectrometry - Espectrometria de Absorção com Chama), realizou-se a média de três determinações, a partir das quais calculou-se seus respectivos desvios padrão. O limite de detecção (LOD) e o limite de quantificação (LOQ) foram calculados a partir das dez leituras dos brancos analíticos para cada elemento, onde o LOD $=3 \times \mathrm{s} / \mathrm{m}$ e $\circ \mathrm{LOQ}=10 \times \mathrm{s} / \mathrm{m}$, no qual considerou-se $\mathrm{s}$ o desvio padrão das medidas dos brancos e $\mathrm{m}$ a inclinação da curva de calibração utilizada para determinação de $\mathrm{Ca}, \mathrm{Mg}, \mathrm{Zn}, \mathrm{Mn}, \mathrm{Fe}$ e Cu.

\section{RESULTADOS E DISCUSSÃO}

$\mathrm{Na}$ tabela 2, são apresentados os dados referentes à curva de calibração para os metais nos chás preparados por infusão e decocção. De acordo com o valor do coeficiente de determinação $\left(R^{2}\right)$ obtido em análise observa-se uma linearidade satisfatória da curva de calibração, contribuindo para obtenção de valores precisos (QUININO et al., s/d).

TABELA 2 - Dados relativos à curva de calibração de Ca, Mg, Zn, Mn, Fe e Cu.

\begin{tabular}{lccccc}
\hline Elemento & $\begin{array}{c}\text { Faixa Linear } \\
\left(\boldsymbol{\mu g} \cdot \mathbf{g}^{-1}\right)\end{array}$ & Equação da Reta & $\mathbf{R}^{2}$ & $\begin{array}{c}\text { LOD } \\
\left(\boldsymbol{\mu g} \cdot \mathbf{g}^{-1}\right)\end{array}$ & $\begin{array}{c}\text { LOQ } \\
\left(\boldsymbol{\mu g} \cdot \mathbf{g}^{-1}\right)\end{array}$ \\
\hline $\mathrm{Ca}$ & $0,0-15$ & $\mathrm{Y}=0,04091 \mathrm{x}+0,0051$ & 0,9993 & 0,227 & 0,757 \\
\hline $\mathrm{Mg}$ & $0,0-1,5$ & $\mathrm{Y}=0,44782 \mathrm{x}+0,0168$ & 0,9970 & 0,157 & 0,523 \\
\hline $\mathrm{Zn}$ & $0,02-2,0$ & $\mathrm{Y}=0,20039 \mathrm{x}+0,0001$ & 0,9994 & $1.10^{-5}$ & $6.10^{-5}$ \\
\hline $\mathrm{Mn}$ & $0,0-10$ & $\mathrm{Y}=0,07414 \mathrm{x}+0,0077$ & 0,9992 & 0,335 & 1,119 \\
\hline $\mathrm{Fe}$ & $0,0-15$ & $\mathrm{Y}=0,02596 \mathrm{x}+0,0006$ & 0,9997 & 1,513 & 5,046 \\
\hline $\mathrm{Cu}$ & $0,0-15$ & $\mathrm{Y}=0,04652 \mathrm{x}+0,0056$ & 0,9993 & $1.10^{-6}$ & $3.10^{-6}$ \\
\hline & \multicolumn{4}{c}{ Fonte: Museu Paraense Emílio Goeldi, Laboratório de absorção atômica, 2017. }
\end{tabular}

Legenda: $R^{2}$ : coeficiente de determinação; LOD: limite de detecção; LOQ: limite de quantificação. 
A tabela 3 apresenta os teores médios e desvios padrão dos resultados das análises das amostras submetidas à digestão (folha seca) e amostras de chá de pariri, por infusão e decocção, dos dois locais de procedência, Mosqueiro e Ver-oPeso.

TABELA 3 - Teor médio e desvio padrão das análises em triplicata para os elementos $\mathrm{Ca}, \mathrm{Mg}, \mathrm{Zn}, \mathrm{Mn}$, $\mathrm{Fe}$ e $\mathrm{Cu}$ nas folhas e nos preparos por infusão e decocção, de amostras de pariri da llha de Mosqueiro e Ver-o-Peso em(mg/L)

\begin{tabular}{|c|c|c|c|c|c|c|}
\hline Elemento & $\begin{array}{l}\text { Digestão } \\
\text { (M.) }\end{array}$ & $\begin{array}{c}\text { Digestão } \\
\text { (V.P.) }\end{array}$ & $\begin{array}{l}\text { Infusão } \\
\text { (M.) }\end{array}$ & $\begin{array}{l}\text { Infusão } \\
\text { (V.P.) }\end{array}$ & $\begin{array}{l}\text { Decocção } \\
\text { (M.) }\end{array}$ & $\begin{array}{c}\text { Decocção } \\
\text { (V.P.) }\end{array}$ \\
\hline $\mathrm{Ca}$ & $\begin{array}{c}3.046,730 \\
\pm \\
144,520\end{array}$ & $\begin{array}{c}691,394 \\
\pm \\
329,333\end{array}$ & $\begin{array}{c}36,7417 \\
\pm \\
12,2708\end{array}$ & $\begin{array}{c}54,7479 \\
\pm \\
12,1964\end{array}$ & $\begin{array}{c}55,2549 \\
\pm \\
4,6069\end{array}$ & $\begin{array}{c}81,1427 \\
\pm \\
12,9135\end{array}$ \\
\hline$\overline{M g}$ & $\begin{array}{c}222,2787 \\
\pm \\
17,9946\end{array}$ & $\begin{array}{c}79,5811 \\
\pm \\
43,7250\end{array}$ & $\begin{array}{c}5,303 \\
\pm \\
1,218\end{array}$ & $\begin{array}{c}10,5038 \\
\pm \\
2,0582\end{array}$ & $\begin{array}{c}8,4105 \\
\pm \\
0,6920\end{array}$ & $\begin{array}{c}13,7740 \\
\pm \\
1,6907\end{array}$ \\
\hline$\overline{Z n}$ & $\begin{array}{c}5,9483 \\
\pm \\
0,3053\end{array}$ & $\begin{array}{c}7,4794 \\
\pm \\
0,6976\end{array}$ & $\begin{array}{c}0,0395 \\
\pm \\
0,0047\end{array}$ & $\begin{array}{c}0,0743 \\
\pm \\
0,0150\end{array}$ & $\begin{array}{c}0,0698 \\
\pm \\
0,0211\end{array}$ & $\begin{array}{c}0,0695 \\
\pm \\
0,0087\end{array}$ \\
\hline$\overline{\mathrm{Mn}}$ & $\overline{<\mathrm{LD}^{\prime}}$ & $\begin{array}{c}0,5697 \\
\pm \\
0,3543\end{array}$ & $<L^{*} D^{*}$ & $\begin{array}{c}0,0782 \\
\pm \\
0,0110\end{array}$ & $\overline{<L D}$ & $\begin{array}{c}0,0687 \\
\pm \\
0,0307\end{array}$ \\
\hline $\mathrm{Fe}$ & $\begin{array}{c}27,5 \\
\pm \\
4,5 \\
\end{array}$ & $\begin{array}{c}54,4 \\
\pm \\
3,1 \\
\end{array}$ & $\begin{array}{l}<\mathrm{LD}^{*} \\
<\mathrm{LD}^{*}\end{array}$ & $\begin{array}{l}<L^{*} \\
<D^{*}\end{array}$ & $\begin{array}{l}<\mathrm{LD}^{*} \\
<\mathrm{LD}^{*}\end{array}$ & $\begin{array}{l}<\mathrm{LD}^{*} \\
<\mathrm{LD}^{*}\end{array}$ \\
\hline$\overline{\mathrm{Cu}}$ & $<\mathrm{LD}^{*}$ & $<\mathrm{LD}^{*}$ & $<\mathrm{LD}^{*}$ & $<\mathrm{LD}^{*}$ & $<\mathrm{LD}^{*}$ & $<\mathrm{LD}^{*}$ \\
\hline
\end{tabular}

Fonte: Museu Paraense Emílio Goeldi, Laboratório de absorção atômica, 2017.

Legenda: $<\mathrm{LD}$ = abaixo do limite de detecção; M.= Mosqueiro; V.P.= Ver-o-Peso.

Comparando as médias, observa-se uma grande disparidade entre a quantidade detectada de cada elemento analisado, em destaque para as amostras submetidas à digestão (folha seca). Para Mengel e Kirkb (2001), esse fenômeno é denominado antagonismo, quando o aumento de um cátion, no meio, pode dificultar a absorção de outro cátion.

Constataram-se, níveis elevados de Ca e Mg. Büll et al. (1998), confirmam que o bom crescimento da planta e absorção de sais minerais, depende não somente de fatores externos (ambientais), como também da existência de outros elementos no solo. Marschner (1995), ratifica que a presença do cátion $\mathrm{Ca}^{+}$ compromete efetivamente a absorção do $\mathrm{Mg}^{+}$.

Nas folhas secas, percebe-se uma concentração maior do Fe em relação ao $Z n$, uma vez que este pode ter sua absorção dificultada devido a interações com alguns nutrientes, entre eles, o Fe (PRADO et al., 2007). A absorção de Zn, pelo solo, também pode ser prejudicada por fatores químicos, como: elevação do pH (acima de 5,5), presença de matéria orgânica, cátions e ânions insolúveis (SHUMAN, 1975).

Segundo Fadigas et al. (2002), o Fe apresenta altas concentrações em solos, logo espera-se encontrar altos teores do mesmo após absorção pelas plantas. Embora o metal tenha um teor maior que o $\mathrm{Zn}$ nas amostras digeridas, o mineral 
não é detectado nos chás. Assim como o $\mathrm{Cu}$, o qual compete com o Zn na absorção pelas plantas (ARZOLLA et al., 1955/1956).

Para Diniz et al. (2013), os valores de Fe obtidos nos digeridos das folhas do pariri, também adquiridos no Ver-o-Peso, estão em torno de $99,1 \mu \mathrm{g} \cdot \mathrm{g}^{-1}$, enquanto que, no trabalho em questão, encontrou-se $54,4 \mu \mathrm{g} \cdot \mathrm{g}^{-1}$. É importante ressaltar, que em ambas as pesquisas não foram encontrados níveis detectáveis do metal nos chás, apenas nos digeridos.

$\mathrm{Na}$ tabela 3, observa-se baixos níveis de Mn para amostras de Mosqueiro e indetectáveis para o Ver-o-Peso. Para Humphries et al. (2006), o teor do mineral nas folhas pode ser alterado devido à presença de infecções de doenças no vegetal, provocadas pela deficiência ou toxicidade de Mn. Outro fator, é a possível elevação do $\mathrm{pH}$ no solo, responsável por diminuir o fornecimento do sal para a planta (MALAVOLTA ; KLIEMANN, 1985; DONADIO et al., 2001).

Nas amostras submetidas a digestão e nos chás, foram notados valores discrepantes dos metais, para os dois locais de procedência, apresentando destaque as triplicatas originadas no mercado. A tabela a seguir demonstra o teor de cada amostra analisada.

TABELA 4 -Valores das triplicatas de cada elemento analisado e seus respectivos locais de procedência, em $\mathrm{mg} / \mathrm{L}$.

\begin{tabular}{|c|c|c|c|c|c|c|}
\hline & $\mathrm{Ca}$ & $\mathrm{Mg}$ & $\mathrm{Zn}$ & $\mathrm{Mn}$ & $\mathrm{Fe}$ & $\mathrm{Cu}$ \\
\hline \multicolumn{7}{|l|}{ Mosqueiro } \\
\hline Digestão 01 & $2.848,5$ & 196,83 & 6,29 & $<L^{*}$ & 26,40 & $<L_{*}^{*}$ \\
\hline Digestão 02 & $3.102,5$ & 235,41 & 5,54 & $<L^{*}$ & 33,49 & $<L_{*}^{*}$ \\
\hline Digestão 03 & $3.189,0$ & 234,59 & 6,00 & $<L_{*}^{*}$ & 22,81 & $<L^{*}$ \\
\hline Infusão 01 & 20,56 & 3,64 & 0,03 & $<L^{*}$ & $<\mathrm{LD}^{*}$ & $<L^{*}$ \\
\hline Infusão 02 & 50,26 & 6,53 & 0,04 & $<L^{*}$ & $<L^{*}$ & $<L^{*}$ \\
\hline Infusão 03 & 39,40 & 5,73 & 0,04 & $<\mathrm{LD}_{*}^{*}$ & $<\mathrm{LD}^{*}$ & $<\mathrm{LD}^{*}$ \\
\hline Decocção 01 & 49,73 & 9,22 & 0,05 & $<\mathrm{LD}^{*}$ & $<L^{*}$ & $<\mathrm{LD}^{*}$ \\
\hline Decocção 02 & 61,00 & 7,52 & 0,09 & $<L_{*}^{*}$ & $<\mathrm{LD}_{*}^{*}$ & $<L_{*}^{*}$ \\
\hline Decocção 03 & 55,02 & 8,48 & 0,05 & $<L^{*}$ & $<L^{*}$ & $<L^{*}$ \\
\hline \multicolumn{7}{|l|}{ Ver-o-Peso } \\
\hline Digestão 01 & 462,4 & 49,53 & 7,72 & 0,72 & 6,29 & $<L^{*}$ \\
\hline Digestão 02 & 454,5 & 47,79 & 8,18 & 0,90 & 133,92 & $<L^{*}$ \\
\hline Digestão 03 & $1.157,1$ & 141,40 & 6,52 & 0,07 & 23,24 & $<L D_{*}^{*}$ \\
\hline Infusão 01 & 51,05 & 9,93 & 0,08 & 0,06 * & $<L_{*}^{*}$ & $<L^{*}$ * \\
\hline Infusão 02 & 42,00 & 8,31 & 0,05 & $<L^{*}$ & $<L^{*}$ & $<L^{*}$ \\
\hline Infusão 03 & 71,18 & 13,26 & 0,08 & 0,08 & $<\mathrm{LD}^{*}$ & $<L D^{*}$ \\
\hline Decocção 01 & 75,00 & 11,91 & 0,06 & 0,04 & $<L^{*}$ & $<L D^{*}$ \\
\hline Decocção 02 & 99,10 & 16,00 & 0,08 & 0,11 & $<\mathrm{LD}_{*}^{*}$ & $<L D_{*}^{*}$ \\
\hline Decocção 03 & 69,31 & 13,40 & 0,06 & 0,04 & $<L^{*}$ & $<L^{*}$ \\
\hline
\end{tabular}

Fonte: Museu Paraense Emílio Goeldi, Laboratório de absorção atômica, 2017.

Legenda: $<$ LD = abaixo do limite de detecção

$\mathrm{De}$ acordo com os dados, os valores das triplicatas para os elementos $\mathrm{Ca}, \mathrm{Mg}$ e Fe, em destaque para o Ver-o-Peso, percebe-se a inexistência de linearidade entre os resultados obtidos. Enquanto que, para as amostras do Distrito de Mosqueiro, encontra-se pouco desnível entre os valores. A tabela a seguir, apresenta o teor, em $\mathrm{mg}$, dos sais minerais em quantidades requeridas pelo organismo, conforme a faixa etária. 
TABELA 5- Dados referentes ao limite máximo de ingestão tolerável, em mg, dos sais de acordo com a faixa etária.

\section{Faixa Etária}

\begin{tabular}{lccccc} 
& $\begin{array}{c}\text { Lactentes } \\
\text { Elementos }\end{array}$ & $\begin{array}{c}\text { Crianças } \\
(0-6 \text { meses })\end{array}$ & $\begin{array}{l}\text { Gestantes } \\
(1-3 \text { anos })\end{array}$ & Lactantes & Adultos \\
\hline $\mathrm{Ca}$ & 300 & 500 & 1200 & 1000 & 1000 \\
$\mathrm{Mg}$ & 36 & 60 & 220 & 270 & 260 \\
$\mathrm{Zn}$ & 2,8 & 4,1 & 11 & 9,5 & 7 \\
$\mathrm{Mn}$ & 0,003 & 1,2 & 2,0 & 2,6 & 2,3 \\
$\mathrm{Fe}$ & 0,27 & 6 & 27 & 15 & 14 \\
$\mathrm{Cu}$ & 200 & 0,7 & 1000 & 1300 & 900 \\
\hline
\end{tabular}

Fonte: ANVISA, 2005.

\section{CONCLUSÕES}

Nas amostras digeridas, de Mosqueiro e Ver-o-Peso, detectaram-se Mn, Zn, $\mathrm{Fe}, \mathrm{Ca}$ e $\mathrm{Mg}$, com destaque para os dois últimos metais. Fato que pode ser explicado, pela volatilização da matéria orgânica, devido à alta temperatura, permanecendo apenas o conteúdo inorgânico. O Cu foi o único a não ser detectado, em função da competição entre alguns elementos, onde a presença de um cátion interfere na absorção de outro, assim como a elevação do $\mathrm{pH}$ pode dificultar a existência do metal no solo.

Nas amostras dos chás, observou-se que Fe e Mn (para Mosqueiro), não apresentaram teores detectáveis. Considerando que a planta requer baixo teor desses micronutrientes, o volume de $50 \mathrm{~mL}$ de água destilada utilizada para preparar o chá ou então o tempo de extração de 5 minutos podem ter sido insuficientes para realizar a extração dos sais. Observou-se, também, uma variação no teor dos metais, destaque para o Ver-o-Peso, devido ao precário controle de informações sobre a planta e solo de origem, já que a maioria das ervas são inerentes da agricultura familiar. Enquanto que, para a llha de Mosqueiro, percebese uma regularidade maior, pois se administrou a escolha da planta, do solo e o tempo de coleta.

Tal cenário comprova a necessidade de uma política de padronização sobre a procedência da planta, por meio da fiscalização da cadeia produtiva que gerencia a comercialização da erva, como o controle sobre a origem de cada amostra e o tempo de coleta, além da criação de uma assistência técnica para as erveiras no aprendizado de boas práticas de manipulação das plantas.

É válido um estudo aprofundado, afim de certificar cientificamente sobre os possíveis efeitos terapêuticos promovidos pelo pariri e então oficializar o uso medicamentoso da planta. Uma alternativa interessante para a utilização da erva, é empregar o consumo do pó do pariri na forma de cápsulas, caso se utilize um extrato seco padronizado e com composição mineral conhecida, principalmente em termos dos metais que apresentaram altos teores.

\section{REFERÊNCIAS}

ABIFISA. Informações sobre fitoterápicos brasileiros. 2007. Disponível em< http://www.abifisa.org.br/> Acessado em 09/01/2017. 
ALBUQUERQUE, J. M. de. Plantas medicinais de uso popular. Brasília ABEAS/MEC. $\quad 105.1989 . \quad$ Disponível em < https://www.bdpa.cnptia.embrapa.br/consulta/busca?b=ad\&biblioteca=vazio\&busca= autoria:\%22ALBUQUERQUE,\%20J.\%20M.\%20de\%22> Acessado em 08/01/2017.

AMOROSO, M. C. M.; GÉLY, A. L. Uso de plantas medicinais por caboclos do baixo Amazonas, Barcarena, PA, Brasil. Boletim do Museu Paraense Emílio Goeldi, Série Botânica, v. 4, n. 1, 47-131, 1988. Disponível em < http://repositorio.museu-goeldi.br/handle/mgoeldi/310> Acessado em 09/01/ 2017.

ANDREWS, G. A.; SMITH, J. Iron metabolism. In: FELDMAN, B. F.; ZINKL, J. G. ; JAIN, N. C. Schalm's veterinary hematology. 5 ed. Philadelphia: Lippincott Willians \& Wilkins, 2000, p. 129-134. Disponível em < https://books.google.com.br/books? id=fVHNBQAAQBAJ\&pg=PA566\&lpg=PA566\&dq $=$ ANDREWS,+ G.+A. ;+SMITH,,+ J.+Iron+metabolism.+In:+FELDMAN,+B.+F.;+ZINKL, + J.+G.+;+JAIN,+N.+C.+Schalm\%E2\%80\%99s+veterinary+hematology.+5+ed.+Phila delphia:+Lippincott+Willians+\%26+Wilkins,+2000,+p.+129-

134.\&source=bl\&ots=uJY3Do2t4_\&sig=ACfU3U0uSLMIHRHxaboStOM4Xz7_GObHF Q\&hl=pt-

BR\&sa $=$ X\&ved=2ahUKEwjZ0uPmxrvhAhXNaFkKHalUAx8Q6AEwCHoECAgQAQ> Acessado em 09/01/2017.

ANVISA. Agência Nacional de Vigilância Sanitária. O "REGULAMENTO TÉCNICO SOBRE A INGESTÃO DIÁRIA". Resolução RDC nํ269, de 22 de setembro de 2005. Disponível em http://portal.anvisa.gov.br/documents/33916/394219/RDC_269_2005.pdf/2e95553ca482-45c3-bdd1-f96162d607b3> Acessado em 09/01/ 2017.

ARZOLLA, J. D. P.; HAAG, H. P.; MALAVOLTA, E. 1955/1956. Nota preliminar sobre a absorção e a translocação do radiozinco. Anais da Escola Superior de Agricultura "Luiz de Queirós", USP (Piracicaba) 12/13: 113 - 120. Disponível em < http://javali.fcav.unesp.br/sgcd/Home/download/pgtrabs/pv/m/3183.pdf> Acessado em 11/01/ 2017.

ASSIS, A. F. S. ANASTÁCIO, F. M. O.; SILVA, M. D. B.; AMARANTE, C. B.; NEVES, P. P. F. G. Determinação do teor de metais em chás de plantas medicinais. Enciclopédia Biosfera, Centro Científico Conhecer - Goiânia, v. 11, n. 21; p. 3396, 2015. 20 Disponível http://www.conhecer.org.br/enciclop/2015b/saude/determinacao\%20do\%20teor\%20 de\%20metais.pdf> Acessado em 11/01/2017.

BARBOSA, W. L. R.; QUIGNARD, E. Projeto Integrado - Departamento de Farmácia / UFPA - Relatório Final de Atividades. Belém/PA, 1989. Disponível em < http://repositorio.ufpa.br/jspui/bitstream/2011/1862/5/Dissertacao_AvaliacaoAtividad eAntimicrobiana.pdf> Acessado em 09/01/2017.

BEHRENS, M. D.; TELLIS, C. J. M.; CHAGAS, M. S. Arrabidaea chica (Humb. \& Bonpl.) B. Verlot (Bignoniaceae). Revista Fitos, Rio de Janeiro, vol. 7 - no 04, p. 236, $2012 . \quad$ Disponível em $<$ https://www.arca.fiocruz.br/bitstream/icict/15126/2/11.pdf> Acessado em 06/05/2019. 
BRASILEIRO, B. G.; PIZZIOLO, V.R.; MATOS, D.S.; GERMANO, A.M.; JAMAL, C.M.; Plantas medicinais utilizadas pela população atendida no "Programa de Saúde da Família", Governador Valadares, MG, Brasil. In: Revista Brasileira de Ciências Farmacêuticas, v. 44, n. 4, 257-261, 2013. Disponível em<https://www.researchgate.net/publication/323434843_Representacoes_Sociais_ do_uso_popular_de_plantas_medicinais_experiencia_em_uma_comunidade_rural> Acessado em 09/01/ 2017.

BORRÁS, M. R. L. Plantas da Amazônia: medicinais ou mágicas? plantas comercializadas no mercado Municipal Adolpho Lisboa. Manaus; Valer/ Governo do Estado do Amazonas, 2003. Disponível em < https://www.amazon.com.br/Plantas-Amaz\%C3\%B4nia-Medicinais-ComercializadasIdentidade/dp/8575120921 > Acessado em 08/01/2017.

BRITES, A. D. Bioquímica da vida: conheça as principais substâncias que compõem os seres vivos. 2014 Disponível em: < https://educacao.uol.com.br/disciplinas/biologia/bioquimica-da-vida-conheca-asprincipais-substancias-que-compoem-os-seres-vivos.htm> Acessado em 09/01/2017.

BÜLL, L.T.; VILLAS BÔAS, R.L. \& NAKAGAWA, J. Variações no balanço catiônico do solo induzidas pela adubação potássica e efeitos na cultura do alho vernalizado. Sci. Agric., 55:157-163,1998. Disponível em < http://www.scielo.br/scielo.php?script=sci_nlinks\&ref=000132\&pid=S0100$0683201000010001600004 \&$ Ing=en $>$ Acessado em 11/01/2017.

CORRÊA, M. P. Dicionário de Plantas uteis no Brasil e das exóticas cultivadas. Rio de Janeiro. 120 Globo, 1978. 6v. Disponível em < https://www.bdpa.cnptia.embrapa.br/consulta/busca?b=ad\&id=814556\&biblioteca=v azio\&busca=autoria:\%22CORREA,\%20M.\%20P.\%22\&qFacets=autoria:\%22CORRE A,\%20M.\%20P.\%22\&sort=\&paginacao=t\&paginaAtual=1 >Acessado em 09/01/2017.

COSTA, P. R. C.; LIMA, E. A. Simpósio Brasileiro de Química e Farmacologia de produtos naturais. Rio de Janeiro, 1989. Disponível em < https://www.academia.edu/27485845/COMPROVA\%C3\%87\%C3\%830_DO_POTE NCIAL_MEDICINAL_DE_Arrabidaea_chica_BIGNONIACEAE> Acessado em 09/01/2017.

CUNHA, A. R.; UMBELINO, B.; CORREIA, M. L.; NEVES, M. F. Efeitos do magnésio sobre a estrutura e a função vascular. Revista Hospital Universitário Pedro Ernesto, v. 10, n. 3. Rio de Janeiro. Jul/Set 2011. Disponível em< http://revista.hupe.uerj.br/detalhe_artigo.asp?id=92> Acessado em 10/01/2017.

DINIZ, V. W. B. ; DANTAS FILHO, H.A. ; MULLER, R.C.S. ; FERNANDES, K.G. Classificação multivariada de ervas medicinais da região amazônica e suas infusões de acordo com sua composição mineral. In:QuímicaNova, v. 36, n. 2, 629-636, 2013.2 Disponível em<http://www.scielo.br/scielo.php?pid=S010040422013000200010\&script=sci_abst ract\&tlng=es $>$ Acessado em 09/01/ 2017.

DONADIO, L. C.; SILVA, J. A. A.; ARAÚJO, P. R. S., PRADO, R. de M. Caramboleira (Averrhoa carambola L.) Jaboticabal: Sociedade Brasileira de 
Fruticultura,

p.

81 ,

2001.

Disponível

em<

http://www.scielo.br/pdf/rbf/v33n4/v33n4a31.pdf> Acessado em 10/01/2017.

FÁVARO, R. M. D.; VANUCCHI, H. Níveis plasmáticos de zinco e antropometria de crianças da periferia de centro urbano do Brasil. Revista Saúde Pública, São Paulo, 24 (1), p. 5-10, 1990. Disponível em < https://www.researchgate.net/publication/250042618_Niveis_plasmaticos_de_zinco_ e_antropometria_de_criancas_da_periferia_de_centro_urbano_no_Brasil> Acessado em 08/01/2017.

FADIGAS, F. S.; AMARAL-SOBRINHO, N. M. B.; MAZUR, N.; ANJOS, L. H. C.; FREIXO, A. A. Concentrações naturais de metais pesados em algumas classes de solos brasileiros. Bragantia, Campinas, v.2, p.151-159, 2002. Disponível em <http://www.scielo.br/scielo.php?script=sci_nlinks\&ref=000106\&pid=S141543662006 00030002400010\&lng=pt> Acessado em 09/01/2017.

$\begin{array}{ll}\text { FOLHA DE SÃO PAULO. Festival Ver-o-Peso, em Belém do Pará, já tem } \\ \text { programação } & 2016 .\end{array}$ em<https://www1.folha.uol.com.br/comida/2016/04/1757329-festival-ver-o-peso-embelem-do-para-ja-tem-programacao-definida.shtml> Acessado em 06/05/2019.

FREIRE, M. S.; SOBRINHO, V. P.; CONCEIÇÃO, G. H. DA. A figura feminina no contexto da Inquisição. Revista de Educação Educere Et Educare. Vol. I nำ I jan./jun. p.53-58, $2006 . \quad$ Disponível em<http://www.uel.br/pos/mestradocomunicacao/wpcontent/uploads/Tend\%C3\%AA ncias-e-arqu\%C3\%A9tipos-nas-resultantes-visuais-de-comportamento-econsumoLORIEN-CRISHNA-ZACARIAS.pdf> Acessado em 09/01/2017.

HUMPHRIES, J. M.; STANGOULIS, J. C. R.; GRAHAM, R. D. Manganese. 12. Handbook of plant nutrition, p. 351-374, 2006. Disponível em< http://www.scielo.br/scielo.php?script=sci_nlinks\&ref=000082\&pid=S0100068320110 $0050002200010 \&$ Ing $=$ pt $>$ Acessado em 12/01/2017.

IBGE. Ilha do Mosqueiro: Belém (PA), 2018. Disponível em: $\leq$ https://biblioteca.ibge.gov.br/index.php/bibliotecacatalogo?view=detalhes\&id=42472 $\geq$ Acessado em: 02/01/2018.

MALAVOLTA,E.; KLIEMANN, H. J. Desordens nutricionais no cerrado. Piracicaba: Potafos, p. 136, 1985. Disponível em< http://scholar.google.com.br/scholar?q=MALAVOLTA,E.\%3B+KLIEMANN,+H.+J.+De sordens+nutricionais+no+cerrado.+Piracicaba:+Potafos, + p. $+136,+1985 . \& \mathrm{hl}=$ pt BR\&as_sdt=0\&as_vis=1\&oi=scholart $>$ Acessado em: 02/01/2018.

MARSCHNER, H. Mineral nutrition of higher plants. 2.ed. London, Academic Press, 1995. 889p. Disponível em< https://doi.org/10.1016/C2009-0-02402-7 > Doi: 10.1016/C2009-0-02402-7. Acessado em: 12/01/2018.

MEIRA FILHO, A. Mosqueiro: ilhas e vilas. Belém: Grafisa, 1978. Disponível em< https://ihgb.org.br/pesquisa/biblioteca/item/7232-mosqueiro-ilhas-e-vilas-augustomeira-filho.html> Acessado em: 12/01/2018.

MENGEL, K. \& KIRKBY, E. Principles of plant nutrition. 5.ed. Dordrecht, Kluwer Academic Publishers, 2001. 849p. Disponível em< 
https://dx.doi.org/10.1093\%2Faob\%2Fmch063>

Doi:

10.1093/aob/mch063.

Acessado em: 12/02/2018.

NUNES, A. J. Mineral cálcio - o que devemos saber?. Revista técnica do farmacêutico. Nova técnica 2. 2013. Disponível em< http://www.anfarmag.org.br/artigos-tecnicos> Acessado em: 12/02/2018.

OLIVEIRA, S. Mercado Ver-o-Peso, Belém: turismo de experiência, 2017. Disponível em: http://www.matraqueando.com.br/mercado-ver-o-peso-belemturismo-deexperiencia $>$. Acessado em: 08/01/ 2018.

PAULETTI, P. M.; BOLZANI, V.S.; YOUNG, M.C.M; Constituintes químicos deArrabidaea chica (Bignoniaceae). In. Química Nova, v. 26, n. 5, 641-643, 2003. Disponível em< http://submission.quimicanova.sbq.org.br/qn/qnol/2003/vol26n5/02AR02096.pdf> Acessado em:12/02/2017.

PRADO, R. M.; ROZANE, D. E.; ROMUALDO, L. M.; MOURO, M. C. Acúmulo de nutrientes na parte aérea do milho cv. P30K75 em função da aplicação de fontes de zinco via semente. Revista de Agricultura, Piracicaba, v.82, p.127-133, 2007.

em< http://www.nutricaodeplantas.agr.br/site/downloads/culturas/milho_acumulonutriente .pdf> Acessado em: 20/04/2018.

PREFEITURA MUNICIPAL DE BELÉM. Portal Mosqueiro. 2015. Disponível em $<$ https://www.google.com/url?sa=i\&source=images\&cd=\&cad=rja\&uact=8\&ved=2ah UKEwi8xtH80YfiAhW8HLkGHR-3AoYQjRx6BAgBEAU\&url=http\%3A\%2F\%2Fcorepa.org.br\%2F2016\%2F05\%2F17\%2Fpontos-turisticos-do-para\%2Fportalmosqueiro\%2F\&psig=AOvVaw2TJR700_o-

ECj11TbnzV4m\&ust=1557256110853704 > Acessado em: 06/05/2019.

QUININO, R. C., REIS, E. A. e BESSEGATO, L. F. O coeficiente e determinação $\mathbf{R}^{2}$ como instrumento didático para avaliar a utilidade de um modelo de regressão linear múltipla. Departamento de Estatística ICEx- UFMG. Departamento de Estatística ICE- UFJF, Brasil. S/d. Disponível em< http://www.est.ufmg.br/portal/arquivos/rts/PD_28102011_Final.pdf> Acessado em: 02/04/2017.

SANDWITH, N. Y.; HUNT, D. R. Bignoniáceas. In: REITZ, R. Flora ilustrada catarinense. 126 FascículoBing. Itajaí: Raulino Reitz, 1974. Disponível em< https://www.google.com/search?rlz=1C1AOHY_pt-

BRBR756BR756\&q=SANDWICH,$+\mathrm{N} .+\mathrm{Y} . ;+\mathrm{HUNT},+\mathrm{D} .+\mathrm{R} .+$ Bignoniaceae $.+\ln :+\mathrm{REITZ}$ ,+R.+Flora+ilustrada+catarinense.+126+Fasc\%C3\%ADculoBing.+ltaja\%C3\%AD:+R aulino+Reitz, +1974 .\&spell=1 \&sa=X\&ved=0ahUKEwjijMH71 LvhAhXGVN8KHdfqBRo QBQgqKAA\&cshid=1554560685924935 > Acessado em: 13/04/2017.

SANTOS, R. L. ; NOBRE, M.S.C ; GUIMARÃES, G.P. ; DANTAS, T.B. ; VIEIRA, K.V.M. et al., ; Contaminação fúngica de plantas medicinais utilizadas em chás. In: Revista de Ciências Farmacêutica Básica Aplicada, v. 34, n. 2, 2008. Acessado em: 02/04/2017.

SPONCHIATO, D. Uma doença chamada púrpura. Disponível em: $<$ https://saude.abril.com.br/medicina/uma-doenca-chamada-purpura/>. Acessado em: 22/11/2017. 
SHUMAN, L. M. The effect of soil properties on zinc adsorption by soils. Proceedings Soil. Science Society of America, Madison, v.39, p.454-458, 1975. Disponível em< doi:10.2136/sssaj1975.03615995003900030025x> Acessado em: 02/04/2017.

TAKEMURA, O. S.; LINUMA, M.; TOSA, H.; MOREIRA, O.A.; NOZAWA, Y.; A flavonefromleaves de Arrabidaea chica f. cuprea. In: Phitochemistry38: 12991300 , 1995.

Disponível

em< https://www.researchgate.net/publication/262620198_Pharmacognostic_analysis_of Arrabidaea_chica_Humb_Bonpl_B_Verlt_Leaves_Bignoniaceae> Acessado em: 02/04/2017.

WIKIMEDIA COMMONS. File: bixina. svg. 2018. Disponível em<https://commons.wikimedia.org/wiki/File:Bixina.svg> Acessado em: 06/05/2019.

WIKIPÉDIA. Ficheiro: genipin. svg. $2015 . \quad$ Disponível em<https://pt.wikipedia.org/wiki/Ficheiro:Genipin.svg > Acessado em: 06/05/2019.

ZORN, B. GARCIA-PINERES, A. J. CASTRO, V.; MURILLO, R.; MORA, G.; MERFORT, I. 3-Desoxyanthocyanidinis from Arrabidaeachica.Phytochemistry, v 56, p. 831-835, 2001. Disponível em< https://www.ncbi.nlm.nih.gov/pubmed/11324913> Acessado em: 13/04/2017. 